\title{
The effects of schedules of reinforcement and gradual or abrupt increases in reward magnitude on resistance to extinction
}

\author{
JACK R. NATION and DONALD DURST \\ Texas A\&M University, College Station, Texas 77843
}

\begin{abstract}
During an initial phase of acquisition training, rats were given 41 trials ( 1 trial/day). The continuous-gradual group received one $45-\mathrm{mg}$ food pellet on each trial during this phase, as did the continuous-abrupt group. Two partial groups, the partial-gradual and partialabrupt groups, were maintained on a 50\% partial reinforcement schedule during this initial phase, with one food pellet as reward on reinforcement trials. In a subsequent 11-day acquisition phase, the continuous-abrupt and partial-abrupt groups were shifted immediately to a 12-pellet reward magnitude administered according to the reinforcement schedule received during Phase 1. The continuous-gradual and partial-gradual groups also continued on their respective reinforcement schedules but experienced gradual increases in reward magnitude up to 12 pellets. Finally, all subjects were given 20 trials of extinction training. The extinction results showed that with partial reinforcement training, gradual increases in magnitude of reward produced greater resistance to extinction than did abrupt increases in reward amount. The theoretical significance of these data was discussed.
\end{abstract}

Capaldi (1980) has recently formulated a theory of instrumental responding that is founded on the principle of reinforcement level. This principle states that reward obtained on a given trial, or series of trials, may influence habit growth differentially, contingent on the context in which the reward is received. One assumption of Capaldi's new model is that conditioning will be greater the greater the discrepancy between expected and obtained reward; that is, habit growth will be optimal when large reward magnitude is experienced in the context of expected small reward. Of particular interest here is the implication of this assumption for matters related to persistence phenomena, most notably those associated with partial reinforcement training. Contained within the sequential model (Capaldi, 1967) of persistence is the notion that partial reinforcement training occasions greater resistance to extinction than continuous reinforcement training by virtue of the development of an association between the memory of nonreward $\left(S^{N}\right)$ and instrumental responding $\left(R_{I}\right)$. Since the habit strength of this hypothesized $S^{N}-R_{I}$ association is alleged to regulate persistence during extinction, it follows from reinforcement level theory that conditions that contribute to a discrepancy between expected and obtained reward and ultimately accelerate the habit growth of $S^{N}-R_{I}$ should enhance resistance to extinction.

The aim of this experiment was to provide empirical support for the above prediction. Specifically, schedules of reinforcement (partial or continuous) were interacted

Requests for reprints should be sent to Jack R. Nation, Department of Psychology, Texas A\&M University, College Station, Texas 77843 . with type of shift upward in reward magnitude (gradual or abrupt) during acquisition training. Subsequently, all subjects (rats) were tested in an extinction phase. One prediction was that regardless of schedule conditions, an abrupt shift upward to a larger magnitude reward should produce greater resistance to extinction than a gradual shift upward to the same magnitude of reward. The superiority of the abrupt shift procedure should obtain, due to a more pronounced discrepancy between expected and obtained reward compared with that occasioned by the gradual shift procedure; that is, with a gradual shift upward, expected and obtained reward should show virtually parallel increases, and habit growth should be at a minimum. The superiority of partial over continuous training was also predicted.

\section{METHOD}

\section{Subjects}

The subjects were 36 experimentally naive male albino rats. The animals were purchased from the Laboratory Supply Company and were approximately 100 days old at the beginning of the experiment. The subjects were randomly assigned to one of four groups $(\mathrm{N}=9 /$ group $)$.

\section{Apparatus}

The apparatus was the same straight-alley maze described in detail in Nation, Wrather, Mellgren, and Spivey (1980, Experiment 2). A teaspoon mounted in the middle of the far end of the goalbox served as the food cup. Start, run, goal, and total times were converted to reciprocals for analysis.

\section{Procedure}

All subjects received 41 acquisition trials during Phase 1 at the rate of 1 trial/day. The continuous-gradual (CG) and continuous-abrupt (CA) groups received one 45-mg food pellet on each trial throughout the first phase of the experiment. 
The partial-gradual (PG) and partial-abrupt (PA) groups received a $50 \%$ partial reinforcement schedule with a repeating schedule of RNRN RNNR NRNR NRRN. On rewarded trials, subjects in each of the partial conditions received one food pellet. All subjects were confined in the goalbox for $30 \mathrm{sec}$ whether or not reward was present, and they were run in squads of four (one from each group), with an intertrial interval of approximately $5 \mathrm{~min}$.

During a subsequent 11-day second phase of acquisition training, subjects in Group CA were given $1245-\mathrm{mg}$ food pellets on each trial at the rate of 1 trial/day. By contrast, Group CG subjects received 1 more pellet on each trial during Phase 2 (i.e., 2 pellets on Trial 1,3 pellets on Trial 2, ..., and 12 pellets on Trial 11). Group PA received 12 pellets on rewarded trials according to the following schedule, RNRNRNRNRNR. Conversely, subjects in Group PG received gradually increasing reward amounts according to this same reinforcement pattern. The reward magnitudes were made to correspond to the magnitudes received by continuous reinforcement control subjects in Group CG. The PG subjects were given 2 pellets on Trial 1, 4 pellets on Trial 3, 6 pellets on Trial 5,8 pellets on Trial 7, 10 pellets on Trial 9, and 12 pellets on Trial 11. Other procedures in the second phase were the same as those used during the initial phase of training.

Finally, all subjects were given 20 trials of extinction training at the rate of 1 trial/day. During extinction, food was not present in the goalbox and subjects were confined for $30 \mathrm{sec}$. Remaining procedures were identical to those used in the earlier phases of the study.

\section{RESULTS}

Since the analyses of start, run, and goal speeds failed to reveal any information not revealed by total speeds, the analyses reported below are restricted to the total speed measure.

\section{Acquisition}

A 4 (conditions: CG, CA, PG, PA) by 2 (days: first day of acquisition, last day of acquisition) repeatedmeasures analysis of variance test performed on the Phase 1 data revealed that all groups did acquire the running response over the course of training. Evidence to this effect came from the report of a significant days effect $[F(1,32)=102.46, p<.01]$. Comparisons indicated that all conditions improved from the first to the last day of acquisition training during Phase 1.

A similar repeated-measures analysis performed on the 11 days of Phase 2 showed a significant Conditions by Days interaction effect $[F(30,320)=1.99, p<.01]$. Individual comparisons of all means indicated that CG subjects were inferior to all other subjects on Day 6. It was further shown that the two abrupt conditions (Groups CA and PA) were inferior on Day 10 relative to the two gradual conditions (Groups CG and PG). At the end of Phase 2 (Day 11), there were no differences among any of the conditions.

\section{Extinction}

The extinction data are shown in Figure 1, in blocks of two trials. The findings from this experiment showed that partial reinforcement during acquisition occasioned

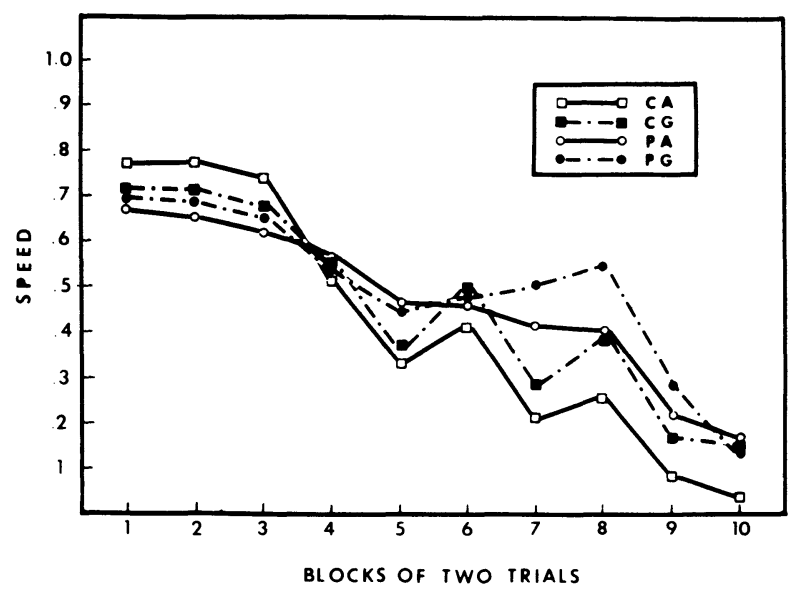

Figure 1. Total speed for extinction in blocks of 2 days (CG $=$ the continuous-gradual group; $C A=$ the continuous-abrupt group; $P G=$ the partial-gradual group; $P A=$ the partial-abrupt group).

greater resistance to extinction than did comparable training with continuous reinforcement, regardless of the type of shift upward (gradual or abrupt). Of greater significance was the finding that under partial reinforcement training a gradual shift upward in reward magnitude produced greater resistance to extinction than did an abrupt shift upward in reward magnitude.

The extinction data were analyzed using a 4 (conditions: CG, CA, PG, PA) by 10 (blocks of two trials) repeated-measures analysis of variance test. Regarding the tests for main effects, only the blocks effect reached an acceptable level for statistical significance $[F(9,288)$ $=73.07, \mathrm{p}<.01]$. Post hoc comparisons using Tukey's test for unconfounded means confirmed what is visually apparent from Figure 1; namely, all subjects declined in performance over trials of extinction. The Conditions by Blocks interaction effect was also found to be significant $[F(27,288)=2.00, p<.01]$. Subsequent analyses performed on cell means showed that on Block 7, Group PG was superior relative to Group PA, which was superior to the remaining two groups (Groups CG and CA). The last two groups were not different from each other on Block 7. On Block 8, Group PG was again superior to Group PA. While there was a trend indicating the superiority of Group CG over Group CA on Block $8(p<.10)$, the only other statistically significant difference on Block 8 was between Groups PA and CA. Remaining blocks showed the convergence of the various groups in late extinction (Blocks 9 and 10).

\section{DISCUSSION}

The findings from this experiment, which show evidence of greater conditioning with gradual as opposed to abrupt shifts upward in reward magnitude, clearly do not support the previously stated predictions derived from interfacing reinforcement level theory (Capaldi, 1980) and the sequential hypothesis (Capaldi, 1967). Since the strength of the $S^{N}-R_{I}$ association is 
believed to be positively related to resistance to extinction, conditions that are favorable to the rapid growth of habit between $S^{N}$ and $R_{I}$ should promote enhanced persistence. According to the principle of reinforcement level, the habit strength of the $S^{N}-R_{I}$ association should have been greater for our partially reinforced subjects experiencing an abrupt shift upward (Group PA) relative to that of our PG subjects. With abrupt shifts upward, the discrepancy between expected and obtained reward should have been large, thus permitting accelerated habit growth during the shift phase (Phase 2). Because partial reinforcement subjects experienced nonreward to reward transitions during this phase, conditioning of the instrumental behavior $\left(R_{I}\right)$ to the memory of nonreward should have occurred (cf. Capaldi, 1967). Consequently, the predicted rapid habit growth should have been specific to $S^{N}-R_{I}$, and persistence should have been increased accordingly. With gradual shifts upward in the partial reinforcement group (Group PG), some strengthening of the $S^{N}-R_{I}$ association should have taken place for the same reasons described above; namely, nonreward-toreward transitions occurred during the shift phase. However, according to the principle of reinforcement level, in this instance habit growth should have been comparatively slow, because under conditions of gradual reward shift, expected and obtained reward should have increased uniformly over the course of training. Since the strength of the $S^{N}-R_{I}$ association should have been reduced in the gradual shift condition relative to the abrupt shift condition, Group PA should have evidenced greater resistance to extinction than Group PG. Our data, of course, showed effects opposite to this prediction.

While our data are at odds with Capaldi's $(1967,1980)$ theoretical forecasts, they are consistent with at least one earlier report on conditioning following gradual or abrupt shifts upward. Nation, Roop, and Dickinson (1976) investigated the influence of gradual or abrupt increases in reward magnitude on the positive contrast phenomenon (see Dunham, 1968, for a review).
In two experiments, it was found that gradual shifts upward in magnitude of reward resulted in more pronounced overshooting (positive contrast) of controls than did abrupt shifts upward. These findings were interpreted as evidence of the superior conditioning associated with the gradual procedure. Unfortunately, there currently exists no theoretical rationale adequately equipped to explain the convergence of data in this area. If indeed gradual increases in reward magnitude do result in greater conditioning than do abrupt increases in reward magnitude, as suggested here and elsewhere, we may be headed for some rather sizable theoretical overhauls in the near future.

\section{REFERENCES}

CAPAldi, E. J. A sequential hypothesis of instrumental learning. In K. W. Spence and J. T. Spence (Eds.), The psychology of learning and motivation: Advances in research and theory (Vol. 1). New York: Academic Press, 1967.

CAPAldi, E. J. Reinforcement level: An expectancy-associative approach to relative reinforcement and nonreinforcement effects. In J. Baerwaldt \& G. McCain (Eds.), The Arlington symposium on learning. Stamford, Conn: Greylock, 1980.

Dunham, P. J. Contrasted conditions of reinforcement: A selective critique. Psychological Bulletin, 1968, 69, 295-315.

Nation, J. R., Roop, S. S., \& Dickinson, R. W. Positive contrast following gradual and abrupt shifts in reward magnitude using delay of reinforcement. Learning and Motivation, 1976, 7, 571-579.

Nation, J. R., Wrather, D. M., Mellgren, R. L., \& Spivey, M. Transfer of the partial reinforcement extinction effect between escape (shock) and appetitive (food) conditioning. Learning and Motivation, 1980, 11, 1-20.

(Received for publication April 15, 1980.) 